\title{
A Systematic Review and Meta-analysis to Determine the Effect of Oral Anticoagulants on Incidence of Dementia in Patients with Atrial Fibrillation
}

\author{
Mingjie Lin ${ }^{1}$, Wenqiang $\mathrm{Han}^{2}$, Jingquan Zhong ${ }^{2}$, and Lin $\mathrm{Wu}^{1}$ \\ ${ }^{1}$ Peking University First Hospital \\ ${ }^{2}$ Shandong University Cheeloo College of Medicine
}

March 12, 2021

\begin{abstract}
Aims: To assess the effect of oral anticoagulant (OAC) administration on incidence of dementia in patients with atrial fibrillation (AF) with Systematic review and meta-analysis in according with the Preferred Reporting Items for Systematic Review and Meta-analysis Protocols. Methods: We systematically searched the electronic databases including Pubmed, Embase, Cochrane library, and ClinicalTrails.gov for relevant articles. The primary outcome was the incidence of dementia. The adjusted risk ratio (RR), odds ratio, or hazard ratio were extracted and pooled by the random-effects models. Subgroup analysis was performed according to the setting observational window. Risk of bias was assessed using the Newcastle-Ottawa Scale, while publication bias was assessed by the Begg's and Egger's tests. Results: Nine studies included in this review (2 prospective and 7 retrospective observational studies, including 613,920 patients). The results presented the significant association between $\mathrm{OAC}$ therapy and the reduced risk of dementia compared with no treatment $(\mathrm{RR}[95 \% \mathrm{CI}]=0.72[0.60,0.86], \mathrm{I} 2=97.2 \% ; \mathrm{P}$ $=0.000$ ). In the subgroup analysis, the pooled RR became statistically non-significant (including four studies, RR [95\%CI] $=0.75[0.51,1.10], \mathrm{I} 2=98.8 \% ; \mathrm{P}=0.000)$. There is no significant risk of bias and publication bias. Conclusions: This study indicated the protective effect of OAC therapy for dementia in patients with AF. However, the results are limited because of high heterogeneity, inconsistent direction of effect in subgroup analysis. Further prospective well-designed study is needed with longer follow-up duration in younger patients.
\end{abstract}

A Systematic Review and Meta-analysis to Determine the Effect of Oral Anticoagulants on Incidence of Dementia in Patients with Atrial Fibrillation

Running head: Anticoagulants, dementia, atrial fibrillation

Mingjie Lin, $\mathrm{PhD}^{1}$, Wenqiang Han, $\mathrm{MD}^{2}$, Jingquan Zhong, $\mathrm{PhD}, \mathrm{MD}^{2,3}$, Lin Wu, BM, MSc, MD ${ }^{1,4,5}$

1. Department of Cardiology, Peking University First Hospital, Beijing, China

2. The Key Laboratory of Cardiovascular Remodeling and Function Research, Chinese Ministry of Education, Chinese National Health Commission and Chinese Academy of Medical Sciences, The State and Shandong Province Joint Key Laboratory of Translational Cardiovascular Medicine, Department of Cardiology, Qilu Hospital, Cheeloo College of Medicine, Shandong University, Jinan, China

3. Department of Cardiology, Qilu Hospital (Qingdao), Cheeloo College of Medicine, Shandong University, Qingdao, China

4. Department of Cardiology, Key Laboratory of Molecular Cardiovascular Science, Ministry of Education, Beijing, China

5. Key Laboratory of Medical Electrophysiology of Ministry of Education, Institute of Cardiovascular Research, Southwest Medical University, Luzhou, China 
Corresponding Author: Lin Wu, Corresponding author. Department of Cardiology, Peking University First Hospital, No. 8 Xishiku Road, Beijing 100034, China; Phone: +86 13716162368; E-mail:linnwu@163.com.

Acknowledgements: We sincerely thank Dr. Ali (Abu Sad Mohammed Arif Ali, Department of Cardiology, Peking University First Hospital, Beijing, China) for correcting the English. This work was supported by the National Natural Science Foundation of China $(81770325,81930105)$. Sponsor is no role in the design, methods, subject recruitment, data collections, analysis and preparation of paper.

Conflict of interest statement: The authors have no conflicts to disclose

\section{Abstract}

Aims:To assess the effect of oral anticoagulant (OAC) administration on incidence of dementia in patients with atrial fibrillation (AF) with Systematic review and meta-analysis in according with the Preferred Reporting Items for Systematic Review and Meta-analysis Protocols.

Methods: We systematically searched the electronic databases including Pubmed, Embase, Cochrane library, and ClinicalTrails.gov for relevant articles. The primary outcome was the incidence of dementia. The adjusted risk ratio (RR), odds ratio, or hazard ratio were extracted and pooled by the random-effects models. Subgroup analysis was performed according to the setting observational window. Risk of bias was assessed using the Newcastle-Ottawa Scale, while publication bias was assessed by the Begg's and Egger's tests.

Results: Nine studies included in this review (2 prospective and 7 retrospective observational studies, including 613,920 patients). The results presented the significant association between OAC therapy and the reduced risk of dementia compared with no treatment $\left(\mathrm{RR}[95 \% \mathrm{CI}]=0.72[0.60,0.86], \mathrm{I}^{2}=97.2 \% ; P=0.000\right)$. In the subgroup analysis, the pooled RR became statistically non-significant (including four studies, RR $\left.[95 \% \mathrm{CI}]=0.75[0.51,1.10], \mathrm{I}^{2}=98.8 \% ; P=0.000\right)$. There is no significant risk of bias and publication bias.

Conclusions: This study indicated the protective effect of OAC therapy for dementia in patients with AF. However, the results are limited because of high heterogeneity, inconsistent direction of effect in subgroup analysis. Further prospective well-designed study is needed with longer follow-up duration in younger patients.

Keywords:atrial fibrillation; anticoagulant; dementia; cognitive impairment

\section{Review criteria}

The inclusion criteria were: i) patients with AF who were [?] 18 years; ii) investigating the effects of OACs on the incidence of dementia; iii) reporting the outcomes as HR, RR, or OR, with 95\%CIs; iv) RCTs or observational studies.

The exclusion criteria were: i) patients with the history of dementia or moderate to severe cognitive dysfunction; ii) only crude or unadjusted HR, RR, or OR; iii) cross-sectional studies; iv) following types of articles: conference abstracts, editorials, letters, expert opinions, case reports, case series, or reviews.

\section{Messages for the clinic}

The results add the further evidences of strict anticoagulation in patients with atrial fibrillation because of its additional potential role on decease risk of incidence of dementia.

The results imply the importance of setting observational window for unveiling the role of anticoagulation on chronic progressive endpoints.

\section{Introduction}

Atrial fibrillation (AF) is one of the most prevalent persistent arrhythmias. ${ }^{1}$ Dementia is defined as deficits in [?]2 cognitive domains that are sufficiently severe to affect activities of daily living, being classified into Alzheimer's disease, vascular dementia, Lewy body dementia, frontotemporal dementia, and other 
dementias. $^{2}$ They both predominantly affect the elderly, and poses significant burden to patients, family, and healthcare systems. Many prospective and retrospective studies have stressed a strong association between $\mathrm{AF}$ and dementia in patients without stroke (risk ratio $(\mathrm{RR})[95 \%$ confidence interval $(\mathrm{CI})]=1.34[1.13$, $1.58])$, in patients with first-ever or recurrent stroke $(\mathrm{RR}[95 \% \mathrm{CI}]=2.7[1.82,4.00])$, and in a general population including with or without a history of stroke $(\mathrm{RR}[95 \% \mathrm{CI}]=1.36[1.23,1.51]) .{ }^{3,4}$ Proposed mechanisms underlying this link involved overt or covert strokes, cerebral hypoperfusion due to reduced cardiac output present in AF rhythm, inflammation, and presence of shared risk factors for AF and dementia. ${ }^{5,} 6$ Among them, the clinical or subclinical embolic brain ischemia is considered to be the main cause of cognitive decline associated with AF. ${ }^{5}$ Thus, it is assumed that anticoagulation may reduce the risk of cognitive decline and dementia in patients with AF.

A number of observational studies investigated the effect of oral anticoagulant (OAC) therapy on incidence of dementia, but the cause-effect relationship is varied. There are two systematic reviews, including cross-sectional studies, investigating the effect of OAC use on the incidence of cognitive impairment and dementia. ${ }^{7}, 8$ One systematic review including 19 studies suggested that, comparing OAC use to no treatment, the pooled odds ratio (OR) had no statistical significance in reducing dementia occurrence $(3$ studies, OR $[95 \% \mathrm{CI}]=0.89[0.47,1.69]) ;^{7}$ while another systematic review including 8 studies suggested that the pooled hazard ratio (HR) had statistical significance in dementia occurrence ( 5 studies, HR[95\% CI] $=0.71[0.69,0.74]){ }^{8}$ The conflicting results likely resulted from the different number of including studies and including cross-sectional studies which could limit confidence in a cause-effect relationship. In order to overcome the main limitation of previous systematic reviews, Mongkhon et al. ${ }^{9}$ conducted a new systematic review which excluded the cross-sectional studies. The pooled adjusted risk ratio $(\mathrm{RR})$ suggested a protective role of OACs on reducing dementia occurrence ( 5 studies, $\mathrm{RR}[95 \% \mathrm{CI}]=0.79[0.67,0.93]$ ). While in sensitivity analyses using leave-one-out method, the result had no statistical significance after omitting studies conducted by Friberg et al. ${ }^{10}$ and Madhavan et al. ${ }^{11}$ The unstable pooled RR was associated with the design of studies and the limitation of small numbers of studies; two included studies ${ }^{12,}{ }^{13}$ had inclusion of patients with moderate to severe cognitive impairment and dementia which may have small benefit from medication interventions; ${ }^{14}$ and only one study had the observational window, i.e. a blank period after the start of observation during which the patients diagnosed of dementia would be excluded to decrease the prescription preference for physicians between OAC use and non-OAC use. Thus, the association between OAC therapy and the incidence of dementia remained controversial. Although there is still a lack of a well-designed randomized controlled trial (RCT) being published, more than 5 studies ${ }^{15-19}$ published since the retrieving data of the latest meta-analysis, and majority of them set the observational window. ${ }^{15-17}$ Therefore, this systematic review and meta-analysis aims to evaluate the effect of the use of OACs on incidence of dementia in patients with AF based on up-to-date evidences; and to evaluate the influence of setting observational window on the pooled RR in subgroup analysis.

\section{Methods}

The present study was performed in accordance with the referred outline in the Cochrane Handbook for Systematic Reviews of Interventions ${ }^{20}$ and the Preferred Reporting Items for Systematic Review and Metaanalysis Protocols (PRISMA-P). ${ }^{21,} 22$ The protocol was prospectively registered in the Prospero International Prospective Register of Systematic Reviews (CRD42020189469).

\section{Data sources and search strategy}

The electronic databases of Pubmed, Embase, Cochrane library, and ClinicalTrails.gov were searched to identify all eligible studies from inception to May 5th, 2020; the search strategy of terms mainly included three parts: atrial fibrillation; anticoagulants or antithrombins or factor Xa inhibitors or dabigatran or DOAC or NOAC or warfarin or vitamin K antagonist*; dementia or Alzheimer disease or cognition disorders or cognitive dysfunction; and their entry terms in MeSH were also used. Reference lists of all included articles and previous meta-analysis articles were manually scanned to supplement the potentially eligible studies. There were no language restrictions. Two authors (Mingjie Lin and Wenqiang Han) independently searched databases and the disagreements were checked by themselves. Full search strategies for all databases are 
presented in Supplementary Table 1.

\section{Study selection and outcome measurement}

Two reviewers (Mingjie Lin and Wenqiang Han) independently screened the titles and abstracts according to the designed criteria; and full articles were obtained for all titles potentially meeting inclusion and exclusion criteria. Endnote Version x9.1 (Clarivate Analytics (US) LLC) was used to manage identified studies and recode the decisions. Reviewers were blinded to each other's decisions. Disagreements between individual judgements were resolved by discussion between themselves and another senior reviewer (Jingquan Zhong). The inclusion criteria were: i) patients with AF who were [?] 18 years; ii) investigating the effects of OACs on the incidence of dementia; iii) reporting the outcomes as HR, RR, or OR, with 95\%CIs; iv) RCTs or observational studies. The exclusion criteria were: i) patients with the history of dementia or moderate to severe cognitive dysfunction; ii) only crude or unadjusted HR, RR, or OR; iii) cross-sectional studies; iv) following types of articles: conference abstracts, editorials, letters, expert opinions, case reports, case series, or reviews. OACs included vitamin $\mathrm{K}$ antagonists (VKAs) and non-vitamin K OACs (NOACs); the control, i.e. Non-OACs included antiplatelet agents or no antithrombotic therapy; dementia included Alzheimer's/Lewy-body/senile/vascular dementia; for overlapping participants, the studies with the longest follow-up and the most detailed information were chosen. The PICOS criteria are presented in Supplementary Table S2.

The primary outcome was the incidence of dementia during the follow-up in AF patients with and without $\mathrm{OAC}$ use. The dementia was diagnosed based on patient narrative, collateral information from families, clinical examination, the standardized cognitive tests, ${ }^{2}$ and diagnostic criteria for dementia including Diagnostic and Statistical Manual of Mental Disorders (DSM)-III, DSM-IV, DSM-V, diagnosis based on international classification of diseases codes (ICD), or United Kingdom (UK) Read codes. ${ }^{9}{ }^{23}$ The additional outcomes were the incidence of dementia in AF patients with: VKAs vs. non-OACs, VKAs vs. NOACs, NOACs vs. non-OACs, and in AF patients with and without OACs use whose $\mathrm{CHA}_{2} \mathrm{DS}_{2}$-VASc score was [?] 2.

\section{Data extraction, strength of evidence assessment, and bias assessment}

Two reviewers (Mingjie Lin and Wenqiang Han) independently extracted the designed variables and data, including first author, year, country, study design, number of patients, age, sex, follow-up time, AF detection, dementia or cognitive deficit definition, $\mathrm{CHADS}_{2}$ or $\mathrm{CHA}_{2} \mathrm{DS}_{2}$-VASc Score, history of stroke, observational window, maximum adjusted covariates, defined OAC use, drugs of OACs, percentage of OACs use, comparison group, adjusted OR/RR/HR with $95 \%$ CIs. The correspondent authors were contacted by e-mail when overall adjusted OR/RR/HR were missing. The study was excluded if the authors did not reply. Another reviewer (Jingquan Zhong) checked the extracted data. Disagreements regarding any extracted data were resolved by discussion among the three reviewers.

Two reviewers (Mingjie Lin and Wenqiang Han) independently assessed the strength of evidence (SOE) and risk of bias. Any disagreements were resolved through discussion. The SOE was evaluated based on five domains: study limitations, directness, consistency, precision, and reporting bias; it was defined as four levels: high, moderate, low, insufficient. ${ }^{24}$ Risk of bias assessment was evaluated with the Cochrane risk of bias tool for $\mathrm{RCTs}^{20}$ and the Newcastle-Ottawa Scale (NOS) for observational studies. ${ }^{25}$ The RCT was classified as low, high, or unclear risk. The observational study with NOS score [?] 8 was defined as high quality.

\section{Statistical analyses}

The included studies with adjusted $\mathrm{OR} / \mathrm{RR} / \mathrm{HR}$ and $95 \% \mathrm{CIs}$ were meta-analyses. The RRs were used as the general effect estimates of studies; HRs were considered comparable to RRs; ${ }^{9}$ ORs were transferred to RRs using the published method. ${ }^{26}$ The RRs and corresponding $95 \%$ CIs were calculated using the DerSimonianLaird random-effects model. Statistical heterogeneity was tested using Cochran Q test (significance level: 0.1 ) and $\mathrm{I}^{2}$ Statistics (>50\%: considerable heterogeneity). ${ }^{27}$ To explore possible sources of heterogeneity, subgroup analyses were performed based on study designs (prospective vs. retrospective studies), a history 
of stroke (patients with a prior stroke only vs. patients with and without prior stroke), observational window (with vs. without); sensitivity analysis was carried out by the leave-one-out method. Publication bias was explored with a funnel plot, and statistically evaluated by the Begg's and Egger's tests, and the trim-and-fill method. All analyses were performed using the STATA 16.0 (StataCorp, College Station, TX, USA), and a $p$-value of $<0.05$ indicated a statistically significant difference.

\section{Results}

\section{Study selection and characteristics}

As shown in Figure 1, of the 1788 records identified in the electronic database, 1435 records were screened based on title and abstract after removing all duplicate records; 48 full-text articles were assessed for eligibility, among which 39 full-text articles were excluded because of overlapping participants, ${ }^{28,}{ }^{29}$ including participants with history of dementia or moderate to severe cognitive dysfunction, ${ }^{12,}{ }^{13}$ and other reasons . Finally, 9 studies were included in the quantitative synthesis. ${ }^{10}, 11,15-19,30,31$

As presented in Supplementary Table S3, of the 9 included studies, 6 were conducted in Europe, 2 in North America, 1 in Asia; all studies were observational; 2 were prospective design, and 7 were retrospective design; a total of 613,920 patients were included; the follow-up time were varied from about 2 to 10 years; 1 study included patients with the history of first-ever ischaemic stroke only, and the others included mixed patients; 4 studies set the observational window varied from 6 months to 4 years. The quality of included studies was high, reflected by a NOS score of 9 (Supplementary Table S4).

\section{The effect of oral anticoagulant use on incidence of dementia}

For OAC use versus non-OAC use, the results including all 9 studies, presented a significant association of the $\mathrm{OAC}$ use and the reduction of incidence of dementia $\left(\mathrm{RR}[95 \% \mathrm{CI}]=0.72[0.60,0.86], \mathrm{I}^{2}=97.2 \% ; P=0.000\right)$ (Figure 2A, Table 1); for studies including patients of $\mathrm{CHA}_{2} \mathrm{DS}_{2}$-VASc score [?] 2, the results including 18,076 patients of 2 studies, displayed the similar trend of the intended outcome $(\mathrm{RR}[95 \% \mathrm{CI}]=0.83[0.72$, $0.97], \mathrm{I}^{2}=0.0 \% ; P=0.021$ ) (Table 1 ). For VKA use vs. non-OAC use, including 514,111 patients of 4 studies, VKA use was significantly associated with reducing the incidence of dementia $(\mathrm{RR}[95 \% \mathrm{CI}]=0.78[0.65$, 0.93], $\mathrm{I}^{2}=93.4 \% ; P=0.006$ ) (Figure $2 \mathrm{~B}$, Table 1 ). For NOAC use vs. non-OAC use, the results including 274,723 patients of 2 studies, had no statistical difference $\left(\mathrm{RR}[95 \% \mathrm{CI}]=0.61[0.27,1.42], \mathrm{I}^{2}=95.7 \% ; P\right.$ $=0.254$ ) (Table 1). For VKA use vs. NOAC use, there was no statistically significant difference of pooled RR synthesized from 2 studies (RR $\left.[95 \% \mathrm{CI}]=0.91[0.75,1.12], \mathrm{I}^{2}=0.0 \% ; P=0.384\right)$ (Table 1).

\section{Subgroup analyses}

As listed in Table 2, in subgroup analyses by study design, the results demonstrated the consistent protective role of OAC use in reducing the incidence of dementia, with the low heterogeneity for prospective studies (Supplementary Figure S1); by patients with the history of prior stroke, the direction of effects were similar; by setting observational window, the pooled RR did not reach the statistically significant level for studies with observational window $\left(\mathrm{RR}[95 \% \mathrm{CI}]=0.75[0.51,1.10], \mathrm{I}^{2}=98.8 \% ; P=0.000\right)$ (Supplementary Figure S2).

\section{Sensitivity analyses}

In the sensitivity analyses using a leave-one-out method, the direction of effects was stable (Supplementary Table S5).

\section{Publication bias and strength of the body of evidence}

The funnel plot presented a relative symmetry (Supplementary Figure S3). There was no obvious publication bias evaluated by Begg's test $(P=0.348)$ and Egger's test $(P=0.770)$, and the pooled RR was unchanged with the trim-and-fill method $(\mathrm{RR}[95 \% \mathrm{CI}]=0.72[0.60,0.86], P=0.000)$.

As listed in Supplementary Table S6, with the GRADE system, we graded SOE for all investigated outcomes as low to moderate, because the plausible confounding factors may exist for the observational design although 
the effect was fully adjusted. The SOE for outcomes between OAC/VKA/NOAC use and no-OAC use was graded as low due to the inconsistency and indirectness.

\section{Discussion}

The present systematic review and meta-analysis found that OAC use significantly decreased the risk of developing dementia in patients with $\mathrm{AF}$ by $28 \%$ compared with no treatment. But in the subgroup analyses with observational window, there is no statistical difference of the risk of dementia between OAC use and no treatment. The high heterogeneity and low SOE may impact the confidence of the results.

The main finding of this study, i.e. the effect of OAC use on the incidence of dementia, is in accordance with the previous two meta-analyses that demonstrated the protective effect of OACs on the incidence of dementia, but high heterogeneity and low SOE were the common limitation. ${ }^{8,} 9$ The present meta-analysis improved the quality of included studies by excluding the cross sectional studies and the dementia-included studies, and updated four studies. The results of the comparison of VKA use and non-OAC use, and VKA use and NOAC use on the risk of dementia, were consistent with the recent meta-analysis. ${ }^{9}$ However, in this study, NOAC use was not associated with the reduced risk of dementia compared with non-OAC use, which was not in line with the general perception. A recent meta-analysis, including 6 RCTs and 2 observational studies, found that NOACs might decrease the risk of cognitive impairment in comparison to VKAs/acetylsalicylic acid, with marginally significant level of synthetic effect for random-effects model (HR $[95 \% \mathrm{CI}]=0.77[0.53$, 1.01], $\left.\mathrm{I}^{2}=39.4 \%\right){ }^{32}$ The results ask for further RCTs for direct comparison of NOACs and VKAs on the risk of dementia. In the latest clinical guideline, ${ }^{1}$ the high risk of stroke, i.e. $\mathrm{CHA}_{2} \mathrm{DS}_{2}$-VASc score [?] 2, is still the strong indication to prescribe OACs. In the present study, we found the similar protective effect of OACs on the incidence of dementia in AF patients with $\mathrm{CHA}_{2} \mathrm{DS}_{2}$-VASc score [?] 2.

It is known that advanced age, shared risk factors, clinical and silent cerebral infarcts may link AF and dementia. $^{2,5}$ One of the leading potential mechanism is the silent cerebral infarcts, which are very common in patients with $\mathrm{AF}$ and are associated with reduced cognitive functioning. The AF-related silent cerebral infarcts are most prevalent in the frontal lobes which play an important role in executive functioning. Therefore, cumulative silent infarcts of the frontal circuit components may contribute to the development of dementia. Considering the antithrombotic role of OACs, it is a reasonable belief that the effective OAC treatment for AF-related infarcts may prevent the occurrence of dementia. This study may support the validity of this potential hypothesis. It is worth mentioning, the pooled $R R$ is not stable in the subgroup analysis with the observational window. In the non-RCT study, physicians prefer to prescribe OACs to patients with good cognitive performance, which may cause the increased diagnosis of dementia within the short time after inclusion. It was seen that dementia cases occurring in the first year during follow-up were more prevalent in non-OAC users. ${ }^{10}$ The observational window may partly prevent the overestimation of the protective effects of OACs. To this end, the pooled RR with the observational window may be closer to the real effect of OACs on the risk of dementia. Therefore, although this meta-analysis indicated a significant association between $\mathrm{OAC}$ use and reduced incidence of dementia, the following limitations of the included studies need to be noted: i) the observational design for all included studies was the primary limitation, which may lead to the different decision of OACs prescription and the different proportion of complications between OAC use and no treatment patients. Although all included studies were adjusted for potential baseline covariates, the maximum adjusted covariates were varied, and some confounding factors may still remain; ii) it is a long progression from the normal cognitive performance to the mild cognitive impairment and the more serious abnormality of dementia. ${ }^{2}$ The long period may result in the varied effects of studies with different follow-up duration and different baseline age. In addition, the worse cognitive status prior to the stage of dementia exactly associated with the no prescription of OACs, which overestimated the positive effect of OACs on risk of dementia. The result of the subgroup analysis with the observational window presented the lower and insignificant pooled RR. Our study excluded the studies included patients with moderate to severe cognitive impairment, while much studies didn't report the data of baseline cognitive status. iii) the adjusted covariates were based on the baseline data, but several covariates like medication management, complications, AF types, were time-varying, which result in the deviation from the true status 
of patients during follow-up. iv) the AF burden was significantly associated with the progression of AFrelated complications, and a few study found that persistent/permanent rather than paroxysmal AF had a high burden to cognitive impairment, ${ }^{33}$ but all included studies didn't report the data of AF types, let alone AF burden.

\section{Strengths and limitations}

The strengths of this systematic review and meta-analysis were: i) applying a comprehensive search strategy; ii) taking the explicit criteria for inclusion of interested studies; iii) using strict statistical methods for analyses; iv) employing the preferred methodology of systematic review and meta-analysis; ${ }^{21,}{ }^{22} \mathrm{v}$ ) performing the test of publication bias with multiple methods.

The limitations of this study were also impossible to ignore, including that: i) the pooled RR was synthesized from the results of observational studies which presented plausible confounding factors decreasing the strength of observed effect. Therefore, the findings should be interpreted with caution; ii) a high degree of heterogeneity was presented, which can't be interpreted from a single study because the sensitivity analyses using a leave-one-out method didn't change the direction of effect. In this study, the origin of heterogeneity may mainly result from the study design, i.e. the low heterogeneity in the prospective studies. However, other factors like races of patients, follow-up duration, the observational window, may importantly impact the heterogenous effects of OAC use on risk of dementia.

\section{Implications for clinical practice and future investigation}

This study with the latest evidence underlined the important role of OACs on the prevention of clinical stroke, but also of the silent cerebral infarcts in patients with AF. While this result was unable to support the use of OACs solely to prevent dementia.

Given the shortages of previously published studies and the characteristic of long-term progression of dementia, future investigation should include: i) well-designed RCTs with longer follow-up in younger patients for direct comparison of OACs and no treatment, ${ }^{34}$ or well-designed study taking comprehensively cognitive test at baseline, adjusting for baseline and time-varying confounding factors, and applying at least 1-year observational window; ii) identifying the cause and effect relationship among AF burden, dementia, and OAC use; iii) evaluating the effect of the percentage of time at risk with access to OACs, rather than the baseline prescription on the risk of dementia; iv) reporting the studies performed in different races.

\section{Conclusions}

OAC therapy was associated with reduced incidence of dementia in patients with AF. However, on account of the high heterogeneity, low SOE, and no statistical result in observational window subgroup analysis, the results were limited. Further well-designed studies are needed to build the reliable link between OAC use and the reduced risk of developing dementia.

\section{References}

[1] Hindricks G, Potpara T, Dagres N, et al. 2020 ESC Guidelines for the diagnosis and management of atrial fibrillation developed in collaboration with the European Association of Cardio-Thoracic Surgery (EACTS). Eur Heart J . 2020.

[2] Dagres N, Chao TF, Fenelon G, et al. European Heart Rhythm Association (EHRA)/Heart Rhythm Society (HRS)/Asia Pacific Heart Rhythm Society (APHRS)/Latin American Heart Rhythm Society (LAHRS) expert consensus on arrhythmias and cognitive function: what is the best practice? Heart Rhythm . 2018;15 : e37-e60.

[3] Islam MM, Poly TN, Walther BA, et al. Association Between Atrial Fibrillation and Dementia: A Meta-Analysis. Front Aging Neurosci . 2019;11 : 305.

[4] Kalantarian S, Stern TA, Mansour M, Ruskin JN. Cognitive impairment associated with atrial fibrillation: a meta-analysis.Ann Intern Med . 2013;158 : 338-346. 
[5] Diener HC, Hart RG, Koudstaal PJ, Lane DA, Lip GYH. Atrial Fibrillation and Cognitive Function: JACC Review Topic of the Week.J Am Coll Cardiol . 2019;73 : 612-619.

[6] Silva R, Miranda CM, Liu T, Tse G, Roever L. Atrial Fibrillation and Risk of Dementia: Epidemiology, Mechanisms, and Effect of Anticoagulation. Front Neurosci . 2019;13 : 18.

[7] Moffitt P, Lane DA, Park H, O'Connell J, Quinn TJ. Thromboprophylaxis in atrial fibrillation and association with cognitive decline: systematic review. Age Ageing . 2016;45 : 767-775.

[8] Cheng W, Liu W, Li B, Li D. Relationship of Anticoagulant Therapy With Cognitive Impairment Among Patients With Atrial Fibrillation: A Meta-Analysis and Systematic Review. J Cardiovasc Pharmacol. 2018;71 : 380-387.

[9] Mongkhon P, Naser AY, Fanning L, et al. Oral anticoagulants and risk of dementia: A systematic review and meta-analysis of observational studies and randomized controlled trials. Neurosci Biobehav Rev . 2019;96 $: 1-9$.

[10] Friberg L, Rosenqvist M. Less dementia with oral anticoagulation in atrial fibrillation. Eur Heart $J$. 2018;39 : 453-460.

[11] Madhavan M, Hu TY, Gersh BJ, et al. Efficacy of Warfarin Anticoagulation and Incident Dementia in a Community-Based Cohort of Atrial Fibrillation. Mayo Clin Proc . 2018;93 : 145-154.

[12] Mavaddat N, Roalfe A, Fletcher K, et al. Warfarin versus aspirin for prevention of cognitive decline in atrial fibrillation: randomized controlled trial (Birmingham Atrial Fibrillation Treatment of the Aged Study). Stroke . 2014;45 : 1381-1386.

[13] Douiri A, McKevitt C, Emmett ES, Rudd AG, Wolfe CD. Long-term effects of secondary prevention on cognitive function in stroke patients. Circulation . 2013;128 : 1341-1348.

[14] Joe E, Ringman JM. Cognitive symptoms of Alzheimer's disease: clinical management and prevention. $B M J$. 2019;367 : 16217 .

[15] Mongkhon P, Fanning L, Lau WCY, et al. Oral anticoagulant and reduced risk of dementia in patients with atrial fibrillation: A population-based cohort study. Heart Rhythm . 2020;17 : 706-713.

[16] Field TS, Weijs B, Curcio A, et al. Incident Atrial Fibrillation, Dementia and the Role of Anticoagulation: A Population-Based Cohort Study. Thromb Haemost . 2019;119 : 981-991.

[17] Nah MA, Lee KS, Hwang TY. Association Between Atrial Fibrillation and the Risk of Dementia in the Korean Elderly: A 10-Year Nationwide Cohort Study. J Prev Med Public Health . 2020;53 : 56-63.

[18] Krawczyk M, Fridman S, Cheng Y, Fang J, Saposnik G, Sposato LA. Atrial fibrillation diagnosed after stroke and dementia risk: cohort study of first-ever ischaemic stroke patients aged 65 or older.Europace . 2019;21 : 1793-1801.

[19] Ding M, Fratiglioni L, Johnell K, et al. Atrial fibrillation, antithrombotic treatment, and cognitive aging: A population-based study. Neurology . 2018;91 : e1732-e1740.

[20] Higgins J, Thomas J, Chandler J, et al. Cochrane Handbook for Systematic Reviews of Interventions version 6.0 (updated July 2019). Cochrane: Cochrane, 2019.

[21] Shamseer L, Moher D, Clarke M, et al. Preferred reporting items for systematic review and meta-analysis protocols (PRISMA-P) 2015: elaboration and explanation. BMJ . 2015;350 : g7647.

[22] Moher D, Shamseer L, Clarke M, et al. Preferred reporting items for systematic review and meta-analysis protocols (PRISMA-P) 2015 statement. Syst Rev . 2015;4 : 1.

[23] Pink J, O'Brien J, Robinson L, Longson D, Guideline C. Dementia: assessment, management and support: summary of updated NICE guidance. BMJ . 2018;361 : k2438. 
[24] Berkman ND, Lohr KN, Ansari MT, et al. Grading the strength of a body of evidence when assessing health care interventions: an EPC update. J Clin Epidemiol . 2015;68 : 1312-1324.

[25] Wells G, Shea B, O'Connell D, et al. The Newcastle-Ottawa Scale (NOS) for assessing the quality of nonrandomised studies in meta-analyses. Ottawa Hospital Research Institute, 2014.

[26] Zhang J, Yu KF. What's the relative risk? A method of correcting the odds ratio in cohort studies of common outcomes. JAMA . 1998;280 : 1690-1691.

[27] Higgins JP, Thompson SG, Deeks JJ, Altman DG. Measuring inconsistency in meta-analyses. BMJ . 2003;327 : 557-560.

[28] Friberg L, Andersson T, Rosenqvist M. Less dementia and stroke in low-risk patients with atrial fibrillation taking oral anticoagulation. Eur Heart J . 2019;40 : 2327-2335.

[29] Kim D, Yang PS, Yu HT, et al. Risk of dementia in stroke-free patients diagnosed with atrial fibrillation: data from a population-based cohort. Eur Heart J . 2019;40 : 2313-2323.

[30] Barber M, Tait RC, Scott J, Rumley A, Lowe GD, Stott DJ. Dementia in subjects with atrial fibrillation: hemostatic function and the role of anticoagulation. J Thromb Haemost . 2004;2 : 1873-1878.

[31] Marzona I, Baviera M, Vannini T, et al. Risk of dementia and death in patients with atrial fibrillation: A competing risk analysis of a population-based cohort. Int J Cardiol . 2016;220 : 440-444.

[32] Zhang C, Gu ZC, Shen L, et al. Non-vitamin K Antagonist Oral Anticoagulants and Cognitive Impairment in Atrial Fibrillation: Insights From the Meta-Analysis of Over 90,000 Patients of Randomized Controlled Trials and Real-World Studies. Front Aging Neurosci . 2018;10 : 258.

[33] Manolis TA, Manolis AA, Apostolopoulos EJ, Melita H, Manolis AS. Atrial Fibrillation and Cognitive Impairment: An Associated Burden or Burden by Association? Angiology . 2020;71 : 498-519.

[34] Rivard L, Khairy P, Talajic M, et al. Blinded Randomized Trial of Anticoagulation to Prevent Ischemic Stroke and Neurocognitive Impairment in Atrial Fibrillation (BRAIN-AF): Methods and Design. Can J Cardiol . 2019;35 : 1069-1077.

Table 1. Summary of findings of the effect of oral anticoagulant therapy on the risk of dementia

\begin{tabular}{llll}
\hline Outcome & Number of studies & Sample size & Pooled RR(95\%CI) \\
\hline OACs vs. non-OAC & 9 & 613920 & $0.717(0.596-0.862)$ \\
OACs vs. non-OAC in patients with $\mathrm{CHA}_{2} \mathrm{DS}_{2}$-VASc Score[?]2 & 2 & 18076 & $0.834(0716-0.973)$ \\
VKA vs. non-OAC & 4 & 514111 & $0.776(0.647-0.930)$ \\
NOACs vs. non-OAC & 2 & 274723 & $0.614(0.266-1.418)$ \\
VKA vs. NOACs & 2 & 220483 & $0.914(0.746-1.120)$ \\
\hline
\end{tabular}

Table 1. Summary of findings of the effect of oral anticoagulant therapy on the risk of dementia (continued)

\begin{tabular}{llllll}
\hline Z-value & P-value & Heterogeneity & Heterogeneity & Heterogeneity & Heterogeneity \\
\hline & & Q statistic & P-value & I2 & $\tau 2$ \\
3.54 & 0.000 & 287.71 & 0.000 & $97.2 \%$ & 0.0649 \\
2.31 & 0.021 & 0.29 & 0.593 & $0.0 \%$ & 0.000 \\
2.74 & 0.006 & 45.50 & 0.000 & $93.4 \%$ & 0.0247 \\
1.14 & 0.254 & 23.05 & 0.000 & $95.7 \%$ & 0.3492 \\
0.87 & 0.384 & 0.15 & 0.703 & $0.0 \%$ & 0.000 \\
\hline
\end{tabular}


Abbreviations: $\mathrm{RR}=$ relative risk, $\mathrm{CI}=$ confident interval, $\mathrm{OACs}=$ oral anticoagulants, VKA=vitamin $\mathrm{K}$ antagonist, NOACs $=$ non-VKA oral anticoagulants, $\mathrm{NA}=$ not applicable

Table 2. Subgroup analyses of the effect of oral anticoagulant therapy on the risk of dementia

\begin{tabular}{llccc}
\hline Subgroup Characteristics & Number of studies & Pooled RR $(95 \% \mathrm{CI})$ & Z-value & P-value \\
\hline Study design & & & & \\
Prospective studies & 2 & $0.531(0.348-0.812)$ & 2.93 & 0.003 \\
$\begin{array}{l}\text { Retrospective studies } \\
\text { A history of stroke }\end{array}$ & 7 & $0.746(0.613-0.907)$ & 2.94 & 0.003 \\
$\begin{array}{l}\text { Patients with and without stroke } \\
\text { Patients with stroke only }\end{array}$ & 8 & $0.726(0.591-0.893)$ & 3.04 & 0.000 \\
Observational window & 1 & $0.650(0.580-0.720)$ & 7.81 & 0.002 \\
Yes & 4 & & & \\
No & 5 & $0.745(0.505-1.100)$ & 1.48 & 0.138 \\
\hline
\end{tabular}

Table 2. Subgroup analyses of the effect of oral anticoagulant therapy on the risk of dementia (continued)

Heterogeneity

\begin{tabular}{llll}
\hline Q statistic & P-value & $\mathrm{I}^{2}$ & $\tau^{2}$ \\
0.64 & 0.425 & $0.0 \%$ & 0.000 \\
285.55 & 0.000 & $97.9 \%$ & 0.0653 \\
286.27 & 0.000 & $97.6 \%$ & 0.0715 \\
NA & NA & NA & NA \\
244.58 & 0.000 & $98.8 \%$ & 0.1514 \\
30.18 & 0.000 & $86.7 \%$ & 0.0216 \\
\hline
\end{tabular}

Abbreviations: $\mathrm{RR}=$ relative risk, $\mathrm{CI}=$ confident interval, $\mathrm{NA}=$ not applicable

\section{Figure legends}

Figure 1. Flow chart of search result

Figure 2. Risk of dementia of patients with atrial fibrillation receiving oral anticoagulant (OAC) and non$\mathrm{OAC}(\mathrm{A})$; risk of dementia of patients with atrial fibrillation receiving vitamin $\mathrm{K}$ antagonists and non-OAC (B). RR: risk ration; CI: confidence interval; OAC: oral anticoagulant; VKA: vitamin K antagonists

Supplementary Figure S1. The subgroup analysis with prospective and retrospective study. RR: risk ration; CI: confidence interval; OAC: oral anticoagulant

Supplementary Figure S2. The subgroup analysis with and without observational window. RR: risk ration; CI: confidence interval; OAC: oral anticoagulant

Supplementary Figure S3. The funnel plot showing a relative symmetry of publication bias. rr: risk ration 


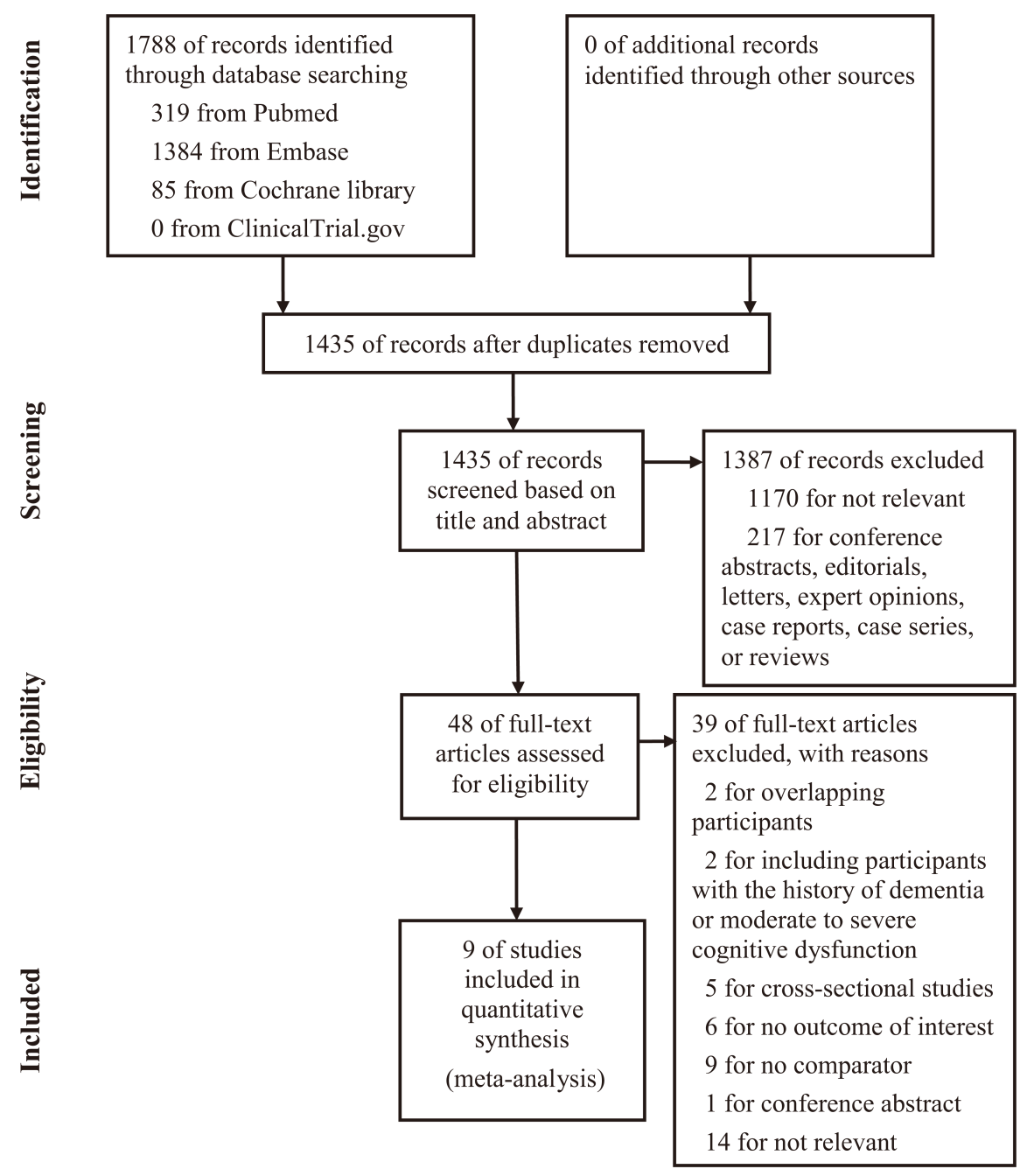


A

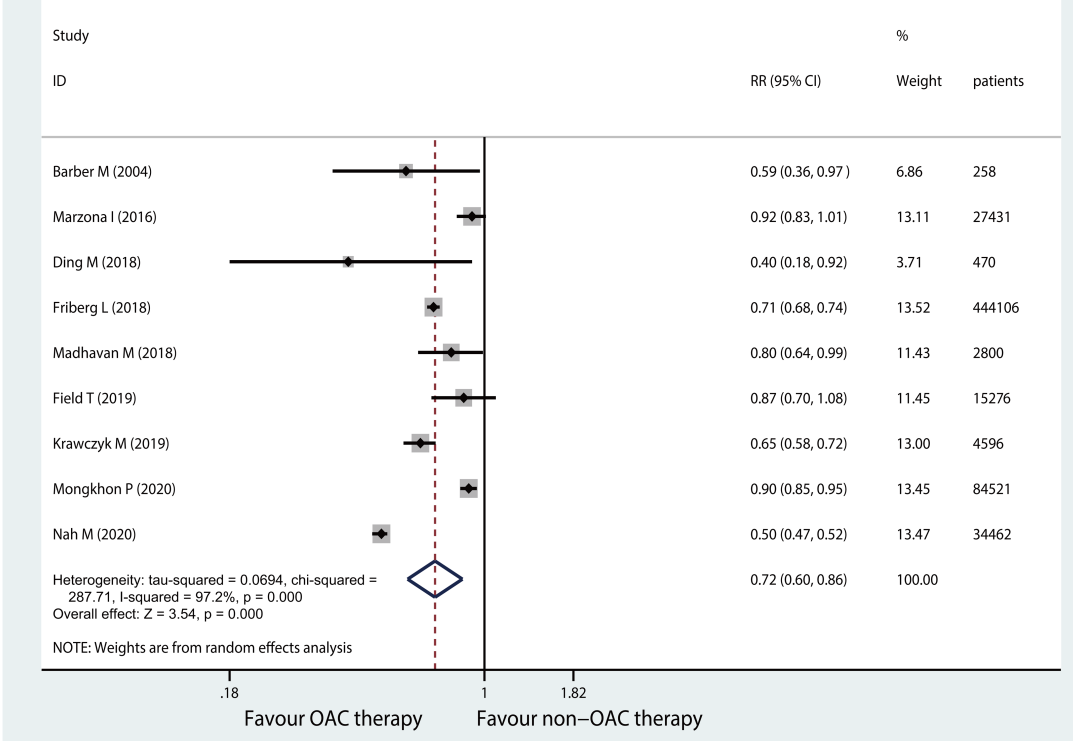

B

Study

RR (95\% Cl) Weight patients

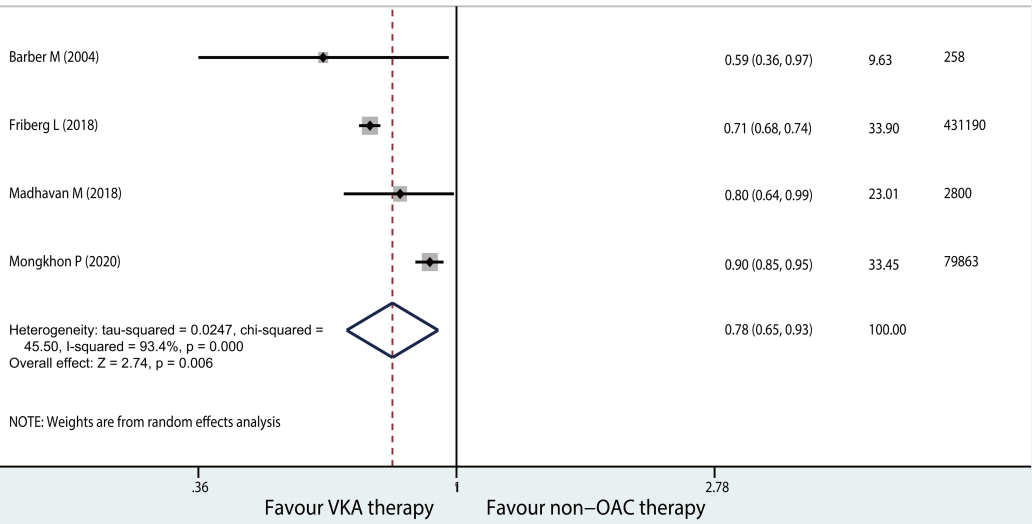

